\title{
Metformin use and cardiovascular outcomes after acute myocardial infarction in patients with type 2 diabetes: a cohort study
}

\author{
Daniel I. Bromage 1,2 (10, Tom R. Godec ${ }^{3}$, Mar Pujades-Rodriguez ${ }^{4}$, Arturo Gonzalez-Izquierdo 5,6,7 , S. Denaxas 5,6,7, \\ Harry Hemingway ${ }^{5,6,7}$ and Derek M. Yellon ${ }^{1 *}$
}

\begin{abstract}
Background: The use of metformin after acute myocardial infarction (AMI) has been associated with reduced mortality in people with type 2 diabetes mellitus (T2DM). However, it is not known if it is acutely cardioprotective in patients taking metformin at the time of AMI. We compared patient outcomes according to metformin status at the time of admission for fatal and non-fatal AMI in a large cohort of patients in England.

Methods: This study used linked data from primary care, hospital admissions and death registry from 4.7 million inhabitants in England, as part of the CALIBER resource. The primary endpoint was a composite of acute myocardial infarction requiring hospitalisation, stroke and cardiovascular death. The secondary endpoints were heart failure (HF) hospitalisation and all-cause mortality.

Results: 4,030 patients with T2DM and incident AMl recorded between January 1998 and October 2010 were included. At AMI admission, $63.9 \%$ of patients were receiving metformin and $36.1 \%$ another oral hypoglycaemic drug. Median follow-up was 343 (IQR: 1-1436) days. Adjusted analyses showed an increased hazard of the composite endpoint in metformin users compared to non-users (HR 1.09 [1.01-1.19]), but not of the secondary endpoints. The higher risk of the composite endpoint in metformin users was only observed in people taking metformin at AMI admission, whereas metformin use post-AMI was associated with a reduction in risk of all-cause mortality $(0.76$ $[0.62-0.93], P=0.009)$.

Conclusions: Our study suggests that metformin use at the time of first AMl is associated with increased risk of cardiovascular disease and death in patients with T2DM, while its use post-AMI might be beneficial. Further investigation in well-designed randomised controlled trials is indicated, especially in view of emerging evidence of cardioprotection from sodium-glucose co-transporter-2 (SGLT2) inhibitors.
\end{abstract}

Keywords: Acute myocardial infarction, Cardioprotection, Cohort studies, Metformin, Outcomes, Type 2 diabetes

\section{Background}

In acute myocardial infarction (AMI), early reperfusion by primary percutaneous coronary intervention (PPCI) is the most effective strategy for reducing infarct size and improving clinical outcome $[1,2]$. However, 30 day allcause mortality following PPCI remains significant and

\footnotetext{
*Correspondence: d.yellon@ucl.ac.uk

${ }^{1}$ The Hatter Cardiovascular Institute, University College London, 67 Chenies Mews, London WC1E 6HX, UK

Full list of author information is available at the end of the article
}

strategies to further improve outcomes are essential [3]. This mortality might be partly explained by the injury inflicted by the therapeutic restoration of blood flow, known as ischaemia-reperfusion injury (IRI), which may account for up to $50 \%$ of final infarct size [4-7].

Diabetes mellitus is a major risk factor for coronary heart disease (CHD), and patients with diabetes and CHD have poor clinical outcomes $[8,9]$. Metformin, an oral antidiabetic of the biguanide class, exerts its effect by increasing gluconeogenesis and increasing peripheral glucose uptake. The UK Prospective Diabetes Study 
(UKPDS) was a large randomised, multicentre trial of glycaemic therapies in patients with newly diagnosed type 2 diabetes mellitus (T2DM) [8]. It showed lower risk of AMI in metformin users than in participants on diet therapy alone. Furthermore, all-cause and cardiovascular mortality was lower in metformin users than in sulfonylurea and insulin users, despite achieving similar glycaemic control. However, the role of metformin in reducing cardiovascular disease remains controversial, with no cardiovascular outcomes achieving statistical significance in a recent meta-analysis [10].

Nonetheless, the potential for metformin to confer acute cardioprotection in AMI is well established in the pre-clinical literature [11-15]. It has been hypothesized that the favorable effects of pre-treatment with metformin in patients with AMI relate to cardioprotection against IRI, independent of its hypoglycaemic actions [14]. However, to our knowledge, no studies have investigated outcomes after AMI in diabetic patients pretreated with metformin, with the exception of mixed results from studies investigating the association between metformin treatment prior to ST-segment elevation AMI (STEMI) and infarct size (defined using serum biomarkers as surrogate measures) and left ventricular (LV) function $[16,17]$. A mortality benefit of pre-treatment with metformin has not been demonstrated in patients with AMI and, using linked electronic health records from the CALIBER resource, which prospectively records medication status, we investigated whether metformin administration is associated with cardioprotection to these patients. Metformin is cheap and widely available, so reducing the ambiguity over its role as a cardioprotective adjunct in AMI could have global relevance [18].

\section{Methods}

\section{Study design and data sources}

This study used linked longitudinal electronic health records from the Clinical Practice Research Datalink (CPRD), Hospital Episode Statistics (HES) and causespecific mortality from the Office for National Statistics (ONS) in England, which are part of the CALIBER resource (https://www.caliberresearch.org). Further details are provided in Additional file 1: Additional methods. This was a prospective cohort study and Additional file 1: Table S1 summarizes the STROBE and RECORD checklists for reporting on observational research [19, 20].

\section{Study population and exposure definition}

The study included all available patients with T2DM experiencing their first fatal or non-fatal AMI (either STEMI or non-STEMI) between January 1998 and October 2010. Diagnoses of T2DM and AMI were identified in CPRD and HES using EHR-derived phenotypes $[21,22]$. Only the earliest recorded AMI across all data sources was considered [21, 23, 24]. Eligibility criteria for study inclusion were no history of AMI prior to the study start date, a minimum of 1 year of follow-up since practice registration and since the date on which the data from their CPRD practice was deemed to be of acceptable quality, and be 18 years of age or above at the time of AMI admission. Metformin exposure status at AMI admission was defined according to whether the patient had at least one prescription within 6 months before the incident AMI. After AMI, if repeat prescriptions were at least 6 monthly, patients were defined as having ongoing drug exposure during follow-up. This was used as a timeupdated covariate in survival analysis.

\section{Baseline characteristics}

To account for recent diabetes severity, it was determined whether a patient was prescribed insulin in 6 months prior to hospital admission. Glycosylated haemoglobin was included, when it was recorded within a year prior to admission, as a marker of glycaemic control. For each patient, data on diagnosed co-morbidities and cardiovascular risk factors were identified in CPRD, HES and ONS [25]. Further details are provided in Additional file 1: Additional methods.

\section{Study endpoints and follow-up}

The primary endpoint was a major adverse cardiac event (MACE), defined as a composite of AMI requiring hospitalisation, stroke and cardiovascular mortality. The secondary endpoints were hospitalisation for heart failure (HF) and all-cause mortality. An a priori subgroup analysis included only survivors of AMI at 30 days postindex AMI (i.e. those who left the GP practice, were 'lost to follow-up' or had an event within 30 days post-AMI were excluded), to distinguish between acute and chronic effects of metformin after AMI. Further details are provided in Additional file 1: Additional methods.

\section{Statistical analyses}

Demographic and baseline characteristics of the study population with and without metformin were compared using the Pearson Chi square test for categorical variables and Student $t$ test for continuous variables. The cumulative probability of each endpoint was calculated using Kaplan-Meier methods and the log rank test was used to examine differences between the treatment groups. Multivariable Cox proportional hazard regression was used to estimate hazard ratios for the effect of metformin use at AMI admission, adjusted for covariates where $\mathrm{P}<0.2$ in the univariable analysis or their inclusion in the models resulted in a change of the estimate of the exposure 
of $>10 \%$, as well as the a priori well established cardiovascular risk factors age, sex, BMI and smoking status at time of index AMI. Further details are provided in Additional file 1: Additional methods.

\section{Sensitivity analyses}

Sensitivity analyses were performed to examine the effect of ongoing metformin use, which has previously been associated with benefit [10]. To do so, we compared patients on metformin at the time of index AMI who had no subsequent prescriptions with those who were never prescribed metformin or had their first prescription after 6 months post-AMI, at which point they were censored). Patients with a metformin prescription within 6 months after first AMI were excluded from this analysis. We also analysed time-varying metformin use for patients who did not have a metformin prescription at AMI admission and within 6 months of AMI, for comparison with previous studies. As an alternative approach, we conducted a propensity score analysis. Propensity scores (the conditional probability that a subject received metformin) were predicted for each subject using baseline covariates in a logistic regression model. All available baseline covariates were considered, but only those that showed evidence of an association with the outcome were used in the final model $[26,27]$. The propensity scores were assessed in each treatment group to ensure balance. Details of the composition of the propensity score model is given in Additional file 1 . The estimated propensity scores were then used to create inverse probability weights for each subject [28]. These weights were used in a Cox proportional hazards regression model to estimate the treatment group effect. Further sensitivity analyses are described in Additional file 1: Additional methods.

\section{Results}

\section{Metformin use and patient baseline characteristics}

Out of 4,703,682 patients in CALIBER, we identified 4030 eligible patients with T2DM and no history of HF or AMI, who had an incident AMI during the study period (Fig. 1). Of them, 2576 (63.9\%) were prescribed metformin at the time of their AMI while 1454 (36.1\%) received an alternative hypoglycaemic drug. The median follow-up time from first AMI (index event) to either death, leaving their GP practice or last date of data collection was 343 (IQR: 1-1436) days.

Patient baseline characteristics according to exposure group are shown in Table 1 . The mean age at index AMI admission was lower for metformin users than for those taking an alternative hypoglycaemic drug (71.3 and 76.1 years, respectively), and there was a lower proportion of women (38.9\% vs. $42.9 \%)$. Overall, co-morbidities and cardiovascular risk factors were similar between the two treatment groups (Table 1). Although more patients in the metformin group had a raised BMI and history of smoking, fewer had a history of peripheral arterial disease (PAD) and abdominal aortic aneurysm (AAA). Importantly, there was no clinically meaningful difference in glycaemic control, indicated by the level of HbA1c, between the two groups (metformin $61.5 \mathrm{mmol} /$

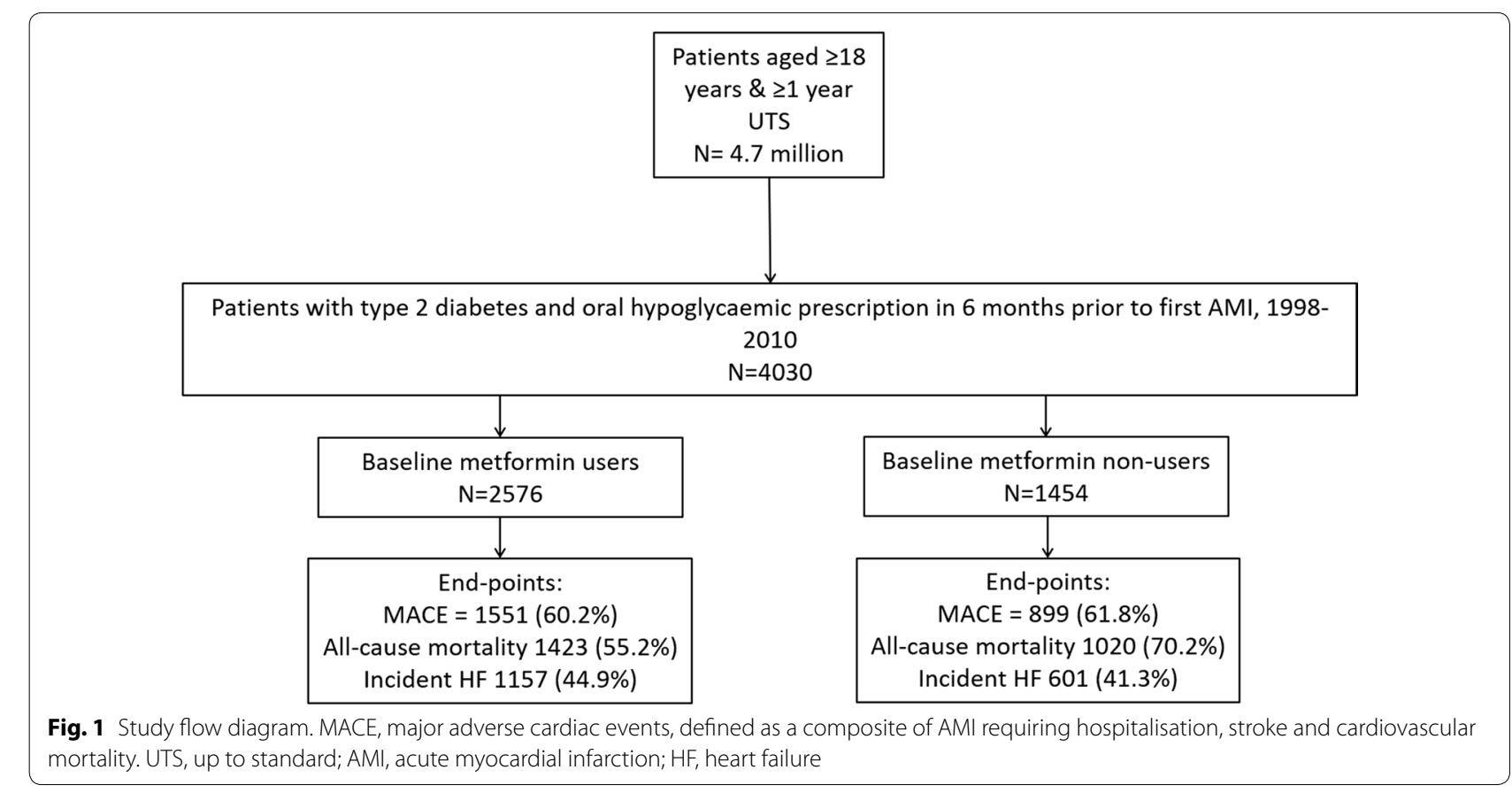


Table 1 Baseline patient characteristics by metformin status, in patients with at least one prescription in 6 months prior to admission with first acute AMI

\begin{tabular}{|c|c|c|}
\hline & Metformin $(\mathrm{N}=2576,63.9 \%)$ & $\begin{array}{l}\text { Other drug } \\
(\mathrm{N}=1454 \text {, } \\
36.1 \%)\end{array}$ \\
\hline Age (years), mean $(S D)^{* *}$ & $71.29(11.2)$ & $76.10(10.9)$ \\
\hline Female, $\mathrm{n}(\%)^{*}$ & $1001(38.9)$ & $624(42.9)$ \\
\hline \multicolumn{3}{|l|}{ IMD, n (\%) } \\
\hline$<8.5$ (Least deprived) & $431(16.8)$ & $252(17.4)$ \\
\hline $8.5-<34.18$ & $1657(64.6)$ & $938(64.8)$ \\
\hline$\geq 34.18$ (Most deprived) & $478(18.6)$ & $258(17.8)$ \\
\hline \multicolumn{3}{|l|}{$\operatorname{BMI}\left(\mathrm{kg} / \mathrm{m}^{2}\right)$, mean $(\mathrm{SD})^{* *}$} \\
\hline$<18.5$ & $23(0.9)$ & $27(2.0)$ \\
\hline $18.5-<25$ & $490(19.8)$ & $388(29.0)$ \\
\hline $25-<30$ & $912(36.8)$ & $535(40.0)$ \\
\hline $30-<35$ & $703(28.4)$ & $275(20.5)$ \\
\hline$\geq 35$ & $352(14.2)$ & $115(8.6)$ \\
\hline \multicolumn{3}{|l|}{ Smoking status, $n(\%)^{*}$} \\
\hline Never-smoker & $1104(44.6)$ & $688(50.4)$ \\
\hline Ex-smoker & $1011(40.8)$ & $493(36.1)$ \\
\hline Current smoker & $363(14.7)$ & $183(13.4)$ \\
\hline Hypertensive, $n(\%)^{*}$ & $2186(84.9)$ & $1178(81.0)$ \\
\hline Systolic blood pressure $(\mathrm{mmHg})$, mean $(\mathrm{SD})^{*}$ & $140.50(23.6)$ & $138.38(21.8)$ \\
\hline $\mathrm{HbA1c}(\mathrm{mmol} / \mathrm{mol})$, mean $(\mathrm{SD})^{* *}$ & $61.48(17.39)$ & $58.99(17.16)$ \\
\hline \multicolumn{3}{|l|}{ Diabetes medication, n (\%) } \\
\hline Sulphonylureas, n (\%)** & $1310(50.9)$ & $1400(96.3)$ \\
\hline Thiazolidinediones, n (\%) & $217(8.4)$ & $146(10.0)$ \\
\hline Acarbose, n (\%) & $69(2.7)$ & $37(2.5)$ \\
\hline DPP4 inhibitors, $n(\%)^{*}$ & $8(0.3)$ & $13(0.9)$ \\
\hline GLP1 agonists, $n(\%)$ & $3(0.1)$ & $2(0.1)$ \\
\hline Meglitinides, n (\%) & $21(0.8)$ & $15(1.0)$ \\
\hline Insulin, $\mathrm{n}(\%)^{* *}$ & $440(17.1)$ & $113(7.8)$ \\
\hline HDL serum cholesterol (mmol/L), mean (SD) & $1.18(0.4)$ & $1.20(0.4)$ \\
\hline Total serum cholesterol (mmol/L), mean (SD)** & $4.43(1.2)$ & $4.58(1.3)$ \\
\hline \multicolumn{3}{|l|}{ History of cardiovascular disease } \\
\hline $\mathrm{HF}, \mathrm{n}(\%)^{*}$ & $652(25.3)$ & $432(29.7)$ \\
\hline $\mathrm{CHD}, \mathrm{n}(\%)$ & $1064(41.3)$ & $593(40.9)$ \\
\hline Ischaemic stroke, n (\%) & $89(3.5)$ & $49(3.4)$ \\
\hline Stroke, n (\%) & $338(13.1)$ & $198(13.6)$ \\
\hline TIA, n (\%) & $218(8.5)$ & $135(9.3)$ \\
\hline$P A D, n(\%)^{*}$ & $472(18.3)$ & $307(21.1)$ \\
\hline AAA, n (\%)* & $441(17.1)$ & $292(20.1)$ \\
\hline
\end{tabular}

Patients with missing data were: 16 for IMD score, 210 for BMI, 188 for smoking status, 91 for systolic blood pressure, 1032 for HbA1C, 988 for HDL cholesterol, and 382 for total cholesterol

$A M I$ acute myocardial infarction, $B M I$ body mass index, IMD index of multiple deprivation, HbA1c haemoglobin A1c, DPP dipeptidyl peptidase, GLP glucagon-like peptide, $H D L$ high density lipoprotein, $H F$ heart failure, $C H D$ coronary heart disease, NOS not otherwise specified, TIA transient ischaemic attack, PAD peripheral arterial disease, $A A A$ abdominal aortic aneurysm

* $\mathrm{P}<0.05 * * \mathrm{P}<0.001$, from tests of difference using $\mathrm{t}$ tests for continuous variables and Chi squared tests for categorical variables

mol vs. other hypoglycemics $59.0 \mathrm{mmol} / \mathrm{mol}$, median time of measurement 96 (IQR 46-168) days prior to index AMI). More patients on metformin had recently been prescribed insulin (17.1\% vs. $7.8 \%)$. Use of other oral hypoglycaemic medication was similar except for sulphonylureas, which were unsurprisingly higher in the non-metformin group (50.9\% vs. $96.3 \%)$. 


\section{Associations between metformin use at AMI admission and endpoints}

In total, 2450 patients had a MACE endpoint during the study period, $1551(60.2 \%)$ in metformin users at the time of their AMI and 899 (61.8\%) in non-users (Table 2). The unadjusted analysis showed no statistically significantly difference between metformin user groups at hospital admission for AMI (HR 0.95 [0.88-1.03], $\mathrm{P}=0.239$, Table 2). In adjusted models, there was strong evidence of an association with the composite endpoint amongst metformin users, but the HR was small (HR 1.09 [1.011.19], $\mathrm{P}=0.034$, Table 2).

The median time to outcome diagnosis was 55 (IQR 0-396) days. Sequential adjustment revealed that adjusting only for age (HR 1.10 [1.01-1.19], $\mathrm{P}=0.033$ ) had the largest impact on the change in the size of the hazard ratio compared to the crude estimate. Further adjustment for other baseline covariates (sex, BMI, prior insulin use, total serum cholesterol, previous stroke and previous TIA) had only small added effects (Additional file 1: Table S2). Furthermore, there was no evidence for an interaction between metformin use and AMI type $(\mathrm{P}=0.274$, Additional file 1: Table S2). The propensity score analysis showed consistent results (HR 1.13 [1.031.23], $\mathrm{P}=0.006$, Table 3) for the composite primary endpoint. This association was driven by an increased hazard of cardiovascular mortality (HR 1.12 [1.00-1.25], $\mathrm{P}=0.044$ ), which was not evident in the main analysis.

With respect to the secondary endpoints, the use of metformin at the time of index AMI was associated
Table 3 Associations between metformin use at hospital admission for AMI and MACE over median follow-up of 343 (IQR 1-1436) days using propensity score analysis

\begin{tabular}{llc}
\hline Endpoint & Hazard ratio $(\mathbf{9 5 \%} \mathbf{C l})$ & P value \\
\hline $\begin{array}{l}\text { Primary endpoint } \\
\text { MACE (composite of cardiovascu- } \\
\quad \text { lar mortality, AMl and stroke) }\end{array}$ & $1.13(1.03-1.23)$ & 0.006 \\
$\begin{array}{l}\text { Components of primary endpoint } \\
\text { Cardiovascular mortality }\end{array}$ & $1.12(1.00-1.25)$ & 0.044 \\
AMl & $1.07(0.95-1.21)$ & 0.273 \\
Stroke & $1.00(0.83-1.21)$ & 0.977 \\
Secondary endpoints & $1.02(0.94-1.11)$ & 0.660 \\
All-cause mortality & $1.18(1.06-1.31)$ & 0.002 \\
HF hospitalisation & & \\
\hline
\end{tabular}

Adjusted for: age at index AMI, sex, ethnicity, BMI, fasted glucose, HbA1c, smoking status, total serum cholesterol, previous $\mathrm{HF}$, previous stroke, previous TIA, previous AAA, previous angina and if ever prescribed insulin prior to index AMI

$A M I$ acute myocardial infarction, $H F$ heart failure

with a trend towards increased risk of HF hospitalisation (HR 1.13 [0.98-1.30], $\mathrm{P}=0.098$, Table 2) but not all-cause mortality (HR 0.97 [0.89-1.04], $\mathrm{P}=0.395$, Table 2). However, in the propensity score analysis, this association with increased HF hospitalisation reached statistical significance (Table 3).

Subgroup analysis restricted to survivors after 30 days post index AMI showed no association between metformin use and MACE (HR 1.06 [0.95-1.17], $\mathrm{P}=0.305$, Additional file 1: Table S3).

Table 2 Associations between metformin use at hospital admission for AMI and MACE over median follow-up of 343 (IQR: 1-1436) days

\begin{tabular}{|c|c|c|c|c|c|c|}
\hline & \multicolumn{2}{|c|}{$\begin{array}{l}\text { Number of patients experiencing event } \\
(\%)\end{array}$} & \multicolumn{2}{|l|}{ Unadjusted } & \multicolumn{2}{|l|}{ Adjusted $^{a}$} \\
\hline & Metformin $(\mathrm{N}=2576)$ & Other $(\mathrm{N}=1454)$ & Hazard ratio $(95 \% \mathrm{Cl})$ & $P$ value & Hazard ratio $(95 \% \mathrm{Cl})$ & $P$ value \\
\hline \multicolumn{7}{|l|}{ Primary endpoint } \\
\hline $\begin{array}{l}\text { MACE (composite of cardio- } \\
\text { vascular mortality, AMI and } \\
\text { stroke) }\end{array}$ & $1551(60.2)$ & $899(61.8)$ & $0.95(0.88-1.03)$ & 0.239 & $1.09(1.01-1.19)$ & 0.034 \\
\hline \multicolumn{7}{|c|}{ Components of primary endpoint } \\
\hline Cardiovascular mortality & $894(34.7 \%)$ & $584(40.2 \%)$ & $0.84(0.76-0.93)$ & 0.001 & $1.06(0.96-1.17)$ & 0.275 \\
\hline AMI & $807(31.3 \%)$ & $433(29.8 \%)$ & $1.01(0.90-1.14)$ & 0.887 & $1.06(0.94-1.20)$ & 0.363 \\
\hline Stroke & $294(11.4 \%)$ & $195(13.4 \%)$ & $0.84(0.70-1.00)$ & 0.051 & $0.99(0.82-1.19)$ & 0.904 \\
\hline \multicolumn{7}{|l|}{ Secondary endpoints } \\
\hline All-cause mortality & $1423(55.2 \%)$ & $1020(70.2 \%)$ & $0.77(0.71-0.83)$ & $<0.001$ & $0.97(0.89-1.04)$ & 0.395 \\
\hline HF hospitalisation & $589(22.9)$ & $313(21.5)$ & $1.01(0.88-1.16)$ & 0.918 & $1.13(0.98-1.30)$ & 0.092 \\
\hline
\end{tabular}

$A M I$ acute myocardial infarction, $H F$ heart failure

a Adjusted for: age at index AMI, sex, smoking status, BMI, prior insulin use, total serum cholesterol, previous stroke, previous TIA 


\section{Sensitivity analyses}

Metformin use at the time of index AMI but not afterwards, compared to no metformin use, was associated with an increased hazard of the primary composite outcome (HR 1.41 [1.28-1.56], $\mathrm{P}<0.001$, Additional file 1: Table S4). This was driven by an increase in the hazard of cardiovascular mortality (HR 1.50 [1.33-1.68], P < 0.001). Patients on metformin at the time of index AMI who had no subsequent prescriptions of this drug had an increased hazard of all-cause mortality (HR 1.31 [1.191.43], $\mathrm{P}<0.001$ ) but the association with HF hospitalisation was not statistically significant (HR 1.12 [0.98-1.45], $\mathrm{P}=0.078$, Additional file 1: Table S4).

In the analysis of time-varying metformin use postAMI in patients who were not on metformin at the time of AMI, the hazard of MACE was similar during periods of metformin use compared to periods of nonuse (HR 0.96 [0.78-1.19], $\mathrm{P}=0.732$ ). However, consistent with previous reports, all-cause mortality was lower during periods of metformin use (HR 0.75 [0.62-0.93], $\mathrm{P}=0.009$ ) in this cohort, as was the hazard of hospitalised HF, although this did not reach statistical significance (HR 0.67 [0.47-1.01], $\mathrm{P}=0.056$ ). There was no evidence of association with cardiovascular mortality (HR 0.88 [0.67-1.15], $\mathrm{P}=0.345$ ). Additional sensitivity analyses are described in Additional file 1: Additional results.

\section{Discussion}

Based on previous basic and clinical studies, we hypothesised that metformin administration would confer cardioprotection in the setting of AMI. We interrogated linked, prospectively recorded, electronic health records of patients with T2DM experiencing their first AMI, using primary care linked to hospital admission and mortality data. This is the first study investigating the association of outcomes after AMI in diabetic patients with metformin pre-treatment. We observed an older cohort, higher rates of previous cardiovascular disease and worse outcomes compared to other studies of AMI in diabetic patients $[29,30]$, indicating that ours is a high-risk cohort.

\section{Metformin use at time of AMI}

Among over 4000 patients identified, the majority were receiving metformin at the time of index AMI, which was associated with worse outcomes with respect to the composite endpoint compared to patients on alternative oral hypoglycaemic agents. This which was driven by cardiovascular mortality and was the case despite similar glycaemic control in the two treatment groups, albeit it with higher rates of insulin prescription in the metformin group. This association was not evident in patients who survived the first month post index AMI, suggesting an acute, non-lasting effect. Interestingly, these findings only applied to patients taking metformin at the time of AMI, whereas post-AMI metformin use was associated with similar or reduced risk (and attenuated the strength of association in the primary analysis), in keeping with previous reports [31]. Taken together and acknowledging potential bias associated with observational studies (including a more severe diabetes phenotype as indicated by a higher use of insulin in metformin users), these findings challenge reports of an acute infarct-sparing role for metformin.

With respect to cardioprotection at the time of AMI, several preclinical studies have demonstrated acute infarct size reduction and improved cardiac function with metformin [11-15]. However, a recently published pre-clinical study of post-conditioning with metformin in pigs found no difference in infarct size or LV function compared to vehicle [32]. Human studies have used biomarkers as a surrogate of infarct size in the context of STEMI [16, 17]. A study of 660 diabetic patients with STEMI showed a reduction of infarct size (according to serum biomarkers) in the metformin group, although the comparator group were treated with diet alone, which may have exaggerated the effect [16]. A propensity score matched analysis of 493 diabetic patients with STEMI showed no association between metformin and infarct size or LV function, although no information on drug treatment of diabetes in the control group was provided [17]. In an analogous, randomized trial of oral metformin, started after primary percutaneous coronary intervention for STEMI in patients without diabetes, no benefit was seen on LV function, MACE, all-cause mortality, or new-onset diabetes [33, 34].

Our data also applies to patients with non-ST-segment elevation AMI (NSTEMI). Despite worse outcomes than patients presenting with STEMI $[35,36]$, there is no evidence regarding the use of metformin in this context. We report an association between metformin use at the time of index AMI, including NSTEMI, and worse outcomes. This was unexpected and justifies further investigation.

We were unable to investigate the reasons for the association with worse outcomes in patients taking metformin at the time of index AMI. Traditionally, metformin is stopped on admission in these patients, due to concerns over lactic acidosis. However, there is a paucity of evidence for this and guidelines from the European Society of Cardiology (ESC) no longer recommend its routine cessation [37-40]. Our findings may also relate to the metformin group being a sicker cohort with worse diabetic phenotypes as indicated by a higher use of insulin in metformin users. In critically ill patients there is a risk of hypoglycaemia-related morbidity associated 
with intensive glucose control with intravenous insulin $[41,42]$, although we did not have data on acute glucose control in our study. In pre-clinical studies, increased myocardial rupture after AMI has been observed with metformin, which has been attributed to increased AMPK-MTOR/PGC- $1 \alpha$-mediated cardiomyocyte autophagy, but we were unable to assess this [43].

\section{Metformin use post-AMI}

Despite these findings, metformin treatment has been shown to be advantageous in several pre-clinical and clinical studies of various cardiovascular diseases [4447]. Beneficial effects on mortality have been reported in patients started on metformin after AMI, independent of its hypoglycaemic actions [31]. This is typically attributed to the prevention of adverse ventricular remodelling [48, 49]. However, these potential effects remain controversial and a recent meta-analysis of randomised trials indicated a non-statistically significant reduction of risk for most outcomes in metformin users, compared to non-users [10]. In the present study, metformin use post-AMI was associated with a lower hazard ratio of MACE, which may support its use in patients at increased risk of cardiovascular disease.

\section{Clinical relevance and further work}

The ESC advocate the use of metformin, despite limited evidence, because of potential safety and economic benefits [50]. The present study is consistent with its use in patients with T2DM but the association with worse outcomes among patients on metformin at the time of their AMI suggests that further investigation in well-designed randomised controlled trials is indicated, especially in view of the evidence and availability of alternatives. Although de novo randomised controlled trials of metformin are unlikely, the present study supports recent assertions that alternative approaches may comprise the inclusion of metformin in factorial trials and full publication of cardiovascular outcome data from previous trials of metformin [10].

\section{Strengths and limitations}

The strengths of this study include, first, the use of three linked electronic data sources to maximise the ascertainment of outcomes. In addition, medication status and baseline characteristics were recorded prospectively, prior to AMI, limiting the possibility of recall bias. Second, the longitudinal study design and the analysis of metformin as a time-varying covariate that consider periods of use and non-use during follow-up. Third, we included patients on alternative hypoglycemics as the control group. This contrasts with similar studies that use a non-diabetic or untreated diabetic patient control arm and risk confounding due to difference in baseline characteristics. The results from the propensity score analysis were consistent, which increases confidence in our findings.

This study has the limitations of observational data, including potential bias because of unmeasured confounding factors (e.g. reperfusion status and indication bias). Therefore, we can only report associations and not causal relationships, and any results must be interpreted with caution, especially in view of clinical experience and economic indications for metformin use. Furthermore, although we accounted for $\mathrm{HbA1c}$ and prior insulin use, we were unable to adjust for other indicators of quality of diabetes management, including microvascular and other macrovascular complications, as these data were unavailable. However, the baseline characteristics of patients in our cohort were similar to each other and to those reported elsewhere [16]. Further strengths and limitations are addressed in Additional file 1: Additional discussion.

\section{Conclusions}

Acknowledging the limitations of observational studies, this study suggests that metformin use at the time of AMI is associated with increased risk of cardiovascular disease and death in patients with T2DM, while its use post-AMI might be beneficial. This may relate to the metformin group being a sicker cohort with worse diabetic phenotypes. However, evidence for metformin in diabetic patients at high cardiovascular risk is mixed and resolving this is central to extracting maximum benefit from this cheap and widely available drug.

\section{Supplementary information}

Supplementary information accompanies this paper at https://doi. org/10.1186/s12933-019-0972-4.

Additional file 1. Supplementary material.

\begin{abstract}
Abbreviations
AAA: abdominal aortic aneurysm; AMl: acute myocardial infarction; BMl: body mass index; CHD: coronary heart disease; CPRD: Clinical Practice Research Datalink; DPP: dipeptidyl peptidase; ESC: European Society of Cardiology; GLP: glucagon-like peptide; HbA1c: haemoglobin A1c; HDL: high density lipoprotein; HES: Hospital episode statistics; HF: heart failure; IMD: index of multiple deprivation; IRI: ischaemia reperfusion injury; LV: left ventricular; ONS: Office for National Statistics; PAD: peripheral arterial disease; PPCl: primary percutaneous coronary intervention; SGLT2: sodium glucose co-transporter-2; STEMI ST: ST-segment elevation myocardial infarction; T2DM: type 2 diabetes mellitus; TIA: transient ischaemic attack.
\end{abstract}

\section{Acknowledgements}

This study is based in part on data from the Clinical Practice Research Datalink obtained under licence from the UK Medicines and Healthcare products Regulatory Agency. The data is provided by patients and collected by the NHS as part of their care and support. The interpretation and conclusions contained in this study are those of the author/s alone. Hospital Episode 
Statistics Copyright @ (2019), re-used with the permission of The Health \& Social Care Information Centre. All rights reserved. The OPCS Classification of Interventions and Procedures, codes, terms and text is Crown copyright (2016) published by Health and Social Care Information Centre, also known as NHS Digital and licensed under the Open Government Licence available at https://www.nationalarchives.gov.uk/doc/open-government-licence/openg overnment-licence.htm. This study was carried out as part of the CALIBER programme (https://www.ucl.ac.uk/healthinformatics/caliber). CALIBER, led from the UCL Institute of Health Informatics, is a research resource consisting of linked electronic health records phenotypes, methods and tools, specialised infrastructure, and training and support. The views expressed are those of the author(s) and not necessarily those of the NHS, the NIHR or the Department of Health.

\section{Authors' contributions}

DB conceived and designed the project and wrote the manuscript. TR performed all analyses. MPR provided significant study design and statistical support and substantively revised the manuscript. AGI acquired and managed the data. SD and HH made substantial contributions to design, data acquisition and interpretation. DY conceived the project and substantively revised the manuscript. All authors read and approved the final manuscript.

\section{Funding}

D.B. has received funding from an MRC Clinical Research Training Fellowship (MR/L002043/1), an NIHR Clinical Lectureship and the Academy of Medical Sciences (SGL020 1087). HH is a National Institute for Health Research (NIHR Senior Investigator). This study is part of the BigData@Heart Consortium that is funded by the Innovative Medicines Initiative-2 Joint Undertaking under grant agreement No. 116074. This Joint Undertaking receives support from the European Union's Horizon 2020 research and innovation programme and EFPIA; it is chaired, by DE Grobbee and SD Anker, partnering with 20 academic and industry partners and ESC. This work is supported by Health Data Research UK, which is funded by the UK Medical Research Council, Engineering and Physical Sciences Research Council, Economic and Social Research Council, Department of Health and Social Care (England), Chief Scientist Office of the Scottish Government Health and Social Care Directorates, Health and Social Care Research and Development Division (Welsh Government), Public Health Agency (Northern Ireland), British Heart Foundation and Wellcome. This study was supported by National Institute for Health Research (RP-PG-040710314), Wellcome Trust (086091/Z/08/Z). This study was supported by the Farr Institute of Health Informatics Research at UCL Partners, from the Medical Research Council, Arthritis Research UK, British Heart Foundation, Cancer Research UK, Chief Scientist Office, Economic and Social Research Council, Engineering and Physical Sciences Research Council, National Institute for Health Research, National Institute for Social Care and Health Research, and Wellcome Trust (MR/K006584/1). This paper represents independent research part funded (AGI, SD) by the National Institute for Health Research (NIHR) Biomedical Research Centre at University College London Hospitals.

\section{Availability of data and materials}

As specified in data sharing agreements, raw datasets are not available. The data that support the findings of this study are available from CPRD (https ://www.cprd.com/) but restrictions apply to the availability of these data, which were used under license for the current study, and so are not publicly available.

\section{Ethics approval and consent to participate}

The study was approved by the MHRA (UK) Independent Scientific Advisory Committee (15_161R), under Section 251 (NHS Social Care Act 2006).

\section{Consent for publication}

Not applicable.

\section{Competing interests}

The authors declare that they have no competing interests.

\section{Author details}

${ }^{1}$ The Hatter Cardiovascular Institute, University College London, 67 Chenies Mews, London WC1E 6HX, UK. ${ }^{2}$ School of Cardiovascular Medicine and Sciences, King's College London British Heart Foundation Centre of Excellence, James Black Centre, 125 Coldharbour Lane, London SE5 9NU, UK. ${ }^{3}$ The
London School of Hygiene \& Tropical Medicine, Keppel St, London WC1E 7HT, UK. ${ }^{4}$ Leeds Institute of Health Sciences, University of Leeds, Clarendon Way, Leeds LS2 9JL, UK. ${ }^{5}$ Institute of Health Informatics, University College London, 222 Euston Road, London NW1 2DA, UK. ${ }^{6}$ Health Data Research UK London, University College London, 222 Euston Road, London NW1 2DA, UK. ${ }^{7}$ The National Institute for Health Research University College London Hospitals Biomedical Research Centre, University College London, 222 Euston Road, London NW1 2DA, UK.

Received: 27 September 2019 Accepted: 29 November 2019 Published online: 09 December 2019

\section{References}

1. Gibson CM. NRMI and current treatment patterns for ST-elevation myocardial infarction. Am Heart J. 2004;148(5 Suppl):S29-33.

2. Keeley EC, Boura JA, Grines CL. Primary angioplasty versus intravenous thrombolytic therapy for acute myocardial infarction: a quantitative review of 23 randomised trials. Lancet. 2003;361(9351):13-20.

3. Pedersen F, Butrymovich V, Kelbaek H, Wachtell K, Helqvist S, Kastrup J, et al. Short- and long-term cause of death in patients treated with primary PCI for STEMI. J Am Coll Cardiol. 2014;64(20):2101-8.

4. Braunwald E, Kloner RA. Myocardial reperfusion: a double-edged sword? J Clin Invest. 1985;76(5):1713-9.

5. Yellon DM, Hausenloy DJ. Myocardial reperfusion injury. N Engl J Med. 2007;357(11):1121-35.

6. Piper HM, Garcia-Dorado D, Ovize M. A fresh look at reperfusion injury. Cardiovasc Res. 1998;38(2):291-300

7. Staat P, Rioufol G, Piot C, Cottin Y, Cung TT, L'Huillier I, et al. Postconditioning the human heart. Circulation. 2005;112(14):2143-8.

8. Turner RC, Millns H, Neil HA, Stratton IM, Manley SE, Matthews DR, et al. Risk factors for coronary artery disease in non-insulin dependent diabetes mellitus: United Kingdom Prospective Diabetes Study (UKPDS: 23). BMJ. 1998;316(7134):823-8.

9. Singh M, Arora R, Kodumuri V, Khosla S, Jawad E. Coronary revascularization in diabetic patients: Current state of evidence. Exp Clin Cardiol. 2011;16(1):16-22.

10. Griffin SJ, Leaver JK, Irving GJ. Impact of metformin on cardiovascular disease: a meta-analysis of randomised trials among people with type 2 diabetes. Diabetologia. 2017;60(9):1620-9.

11. Bhamra GS, Hausenloy DJ, Davidson SM, Carr RD, Paiva M, Wynne AM, et al. Metformin protects the ischemic heart by the Akt-mediated inhibition of mitochondrial permeability transition pore opening. Basic Res Cardiol. 2008;103(3):274-84.

12. Paiva M, Riksen NP, Davidson SM, Hausenloy DJ, Monteiro P, Goncalves L, et al. Metformin prevents myocardial reperfusion injury by activating the adenosine receptor. J Cardiovasc Pharmacol. 2009;53(5):373-8.

13. Paiva MA, Goncalves LM, Providencia LA, Davidson SM, Yellon DM, Mocanu MM. Transitory activation of AMPK at reperfusion protects the ischaemic-reperfused rat myocardium against infarction. Cardiovasc Drugs Ther. 2010;24(1):25-32.

14. Whittington $\mathrm{HJ}$, Hall AR, McLaughlin CP, Hausenloy DJ, Yellon DM, Mocanu MM. Chronic metformin associated cardioprotection against infarction: not just a glucose lowering phenomenon. Cardiovasc Drugs Ther. 2013;27(1):5-16.

15. Hesen NA, Riksen NP, Aalders B, Brouwer MA, Ritskes-Hoitinga M, El Messaoudi $S$, et al. A systematic review and meta-analysis of the protective effects of metformin in experimental myocardial infarction. PLOS ONE. 2017;12(8):e0183664.

16. Lexis CP, Wieringa WG, Hiemstra B, van Deursen VM, Lipsic E, van der Harst $P$, et al. Chronic metformin treatment is associated with reduced myocardial infarct size in diabetic patients with ST-segment elevation myocardial infarction. Cardiovasc Drugs Ther. 2014;28(2):163-71.

17. Basnet S, Kozikowski A, Makaryus AN, Pekmezaris R, Zeltser R, Akerman $M$, et al. Metformin and myocardial injury in patients with diabetes and ST-segment elevation myocardial infarction: a propensity score matched analysis. J Am Heart Assoc. 2015;4(10):e002314.

18. Bromage DI, Yellon DM. The pleiotropic effects of metformin: time for prospective studies. Cardiovasc Diabetol. 2015;14:109. 
19. von Elm E, Altman DG, Egger M, Pocock SJ, Gotzsche PC, Vandenbroucke JP, et al. Strengthening the Reporting of Observational Studies in Epidemiology (STROBE) statement: guidelines for reporting observational studies. BMJ. 2007;335(7624):806-8.

20. Benchimol El, Smeeth L, Guttmann A, Harron K, Moher D, Petersen I, et al. The REporting of studies Conducted using Observational Routinely-collected health Data (RECORD) statement. PLoS Med. 2015;12(10):e1001885.

21. Dinesh Shah A, Langenberg C, Rapsomaniki E, Denaxas S, Pujades-Rodriguez $\mathrm{M}$, Gale $C P$, et al. Type 2 diabetes and incidence of a wide range of cardiovascular diseases: a cohort study in 1.9 million people. Lancet. 2015;385(Suppl 1):S86.

22. George J, Rapsomaniki E, Pujades-Rodriguez M, Shah AD, Denaxas S, Herrett $\mathrm{E}$, et al. How does cardiovascular disease first present in women and men? Incidence of 12 cardiovascular diseases in a contemporary cohort of 1,937,360 people. Circulation. 2015;132(14):1320-8.

23. Herrett E, Shah AD, Boggon R, Denaxas S, Smeeth L, van Staa T, et al. Completeness and diagnostic validity of recording acute myocardial infarction events in primary care, hospital care, disease registry, and national mortality records: cohort study. BMJ. 2013;346:f2350.

24. Rapsomaniki E, Timmis A, George J, Pujades-Rodriguez M, Shah AD, Denaxas $\mathrm{S}$, et al. Blood pressure and incidence of twelve cardiovascular diseases: lifetime risks, healthy life-years lost, and age-specific associations in 1.25 million people. Lancet. 2014;383(9932):1899-911.

25. Pujades-Rodriguez M, Timmis A, Stogiannis D, Rapsomaniki E, Denaxas $\mathrm{S}$, Shah $\mathrm{A}$, et al. Socioeconomic deprivation and the incidence of 12 cardiovascular diseases in 1.9 million women and men: implications for risk prediction and prevention. PLOS ONE. 2014;9(8):e104671.

26. Austin PC. The use of propensity score methods with survival or time-toevent outcomes: reporting measures of effect similar to those used in randomized experiments. Stat Med. 2014;33(7):1242-58.

27. Austin PC. An introduction to propensity score methods for reducing the effects of confounding in observational studies. Multivar Behav Res. 2011;46(3):399-424.

28. Rosenbaum PR, Rubin DB. The central role of the propensity score in observational studies for causal effects. Biometrika. 1983;70(1):41-55.

29. Otter W, Kleybrink S, Doering W, Standl E, Schnell O. Hospital outcome of acute myocardial infarction in patients with and without diabetes mellitus. Diabet Med. 2004;21 (2):183-7.

30. Malmberg K, Ryden L, Wedel H, Birkeland K, Bootsma A, Dickstein K, et al. Intense metabolic control by means of insulin in patients with diabetes mellitus and acute myocardial infarction (DIGAMI 2): effects on mortality and morbidity. Eur Heart J. 2005;26(7):650-61.

31. Mellbin LG, Malmberg K, Norhammar A, Wedel H, Ryden L, Investigators D. Prognostic implications of glucose-lowering treatment in patients with acute myocardial infarction and diabetes: experiences from an extended follow-up of the Diabetes Mellitus Insulin-Glucose Infusion in Acute Myocardial Infarction (DIGAMI) 2 Study. Diabetologia. 2011;54(6):1308-17.

32. Techiryan G, Weil BR, Palka BA, Canty JM Jr. Effect of intracoronary metformin on myocardial infarct size in swine. Circ Res. 2018;123(8):986-95.

33. Lexis CP, van der Horst IC, Lipsic E, Wieringa WG, de Boer RA, van den Heuvel AF, et al. Effect of metformin on left ventricular function after acute myocardial infarction in patients without diabetes: the GIPS-III randomized clinical trial. JAMA. 2014;311(15):1526-35.

34. Hartman MHT, Prins JKB, Schurer RAJ, Lipsic E, Lexis CPH, van der Horst-Schrivers ANA, et al. Two-year follow-up of 4 months metformin treatment vs. placebo in ST-elevation myocardial infarction: data from the GIPS-III RCT. Clin Res Cardiol. 2017;106(12):939-46.

35. Szummer $K$, Wallentin $L$, Lindhagen $L$, Alfredsson J, Erlinge D, Held C, et al. Relations between implementation of new treatments and improved outcomes in patients with non-ST-elevation myocardial infarction during the last 20 years: experiences from SWEDEHEART registry 1995 to 2014. Eur Heart J. 2018;39(42):3766-76. https://doi.org/10.1093/eurheartj/ ehy554.
36. Szummer K, Wallentin L, Lindhagen L, Alfredsson J, Erlinge D, Held C, et al. Improved outcomes in patients with ST-elevation myocardial infarction during the last 20 years are related to implementation of evidence-based treatments: experiences from the SWEDEHEART registry 1995-2014. Eur Heart J. 2017:38(41):3056-65.

37. Tahrani AA, Varughese GI, Scarpello JH, Hanna FW. Metformin, heart failure, and lactic acidosis: is metformin absolutely contraindicated? BMJ. 2007;335(7618):508-12.

38. Khurana R, Malik IS. Metformin: safety in cardiac patients. Heart. 2010;96(2):99-102.

39. Roffi M, Patrono C, Collet JP, Mueller C, Valgimigli M, Andreotti F, et al. 2015 ESC Guidelines for the management of acute coronary syndromes in patients presenting without persistent ST-segment elevation: Task Force for the Management of Acute Coronary Syndromes in Patients Presenting without Persistent ST-Segment Elevation of the European Society of Cardiology (ESC). Eur Heart J. 2016;37(3):267-315.

40. Ibanez B, James S, Agewall S, Antunes MJ, Bucciarelli-Ducci C, Bueno $H$, et al. 2017 ESC Guidelines for the management of acute myocardial infarction in patients presenting with ST-segment elevation: The Task Force for the management of acute myocardial infarction in patients presenting with ST-segment elevation of the European Society of Cardiology (ESC). Eur Heart J. 2018;39(2):119-77.

41. Investigators N-SS, Finfer S, Chittock DR, Su SY, Blair D, Foster D, et al. Intensive versus conventional glucose control in critically ill patients. N Engl J Med. 2009;360(13):1283-97.

42. Senthinathan A, Kelly V, Dzingina M, Jones D, Baker M, Longson D, et al. Hyperglycaemia in acute coronary syndromes: summary of NICE guidance. BMJ. 2011;343:d6646.

43. Hua J, Liu Z, Liu Z, An D, Lai W, Zhan Q, et al. Metformin increases cardiac rupture after myocardial infarction via the AMPK-MTOR/PGC-1alpha signaling pathway in rats with acute myocardial infarction. Med Sci Monit. 2018;24:6989-7000.

44. Slater RE, Strom JG, Methawasin M, Liss M, Gotthardt M, Sweitzer N, et al. Metformin improves diastolic function in an HFpEF-like mouse model by increasing titin compliance. J Gen Physiol. 2019;151(1):42-52.

45. Khan SZ, Rivero M, Nader ND, Cherr GS, Harris LM, Dryjski ML, et al. Metformin is associated with improved survival and decreased cardiac events with no impact on patency and limb salvage after revascularization for peripheral arterial disease. Ann Vasc Surg. 2019;55:63-77.

46. Mary A, Hartemann A, Liabeuf S, Aubert CE, Kemel S, Salem JE, et al. Association between metformin use and below-the-knee arterial calcification score in type 2 diabetic patients. Cardiovasc Diabetol. 2017;16(1):24.

47. Wilmanns JC, Pandey R, Hon O, Chandran A, Schilling JM, Forte E, et al. Metformin intervention prevents cardiac dysfunction in a murine model of adult congenital heart disease. Mol Metab. 2019;20:102-14.

48. Gundewar S, Calvert JW, Jha S, Toedt-Pingel I, Ji SY, Nunez D, et al. Activation of AMP-activated protein kinase by metformin improves left ventricular function and survival in heart failure. Circ Res. 2009;104(3):403-11.

49. Cittadini A, Napoli R, Monti MG, Rea D, Longobardi S, Netti PA, et al. Metformin prevents the development of chronic heart failure in the SHHF rat model. Diabetes. 2012;61(4):944-53.

50. Piepoli MF, Hoes AW, Agewall S, Albus C, Brotons C, Catapano AL, et al. 2016 European Guidelines on cardiovascular disease prevention in clinical practice: The Sixth Joint Task Force of the European Society of Cardiology and Other Societies on Cardiovascular Disease Prevention in Clinical Practice (constituted by representatives of 10 societies and by invited experts)Developed with the special contribution of the European Association for Cardiovascular Prevention \& Rehabilitation (EACPR). Eur Heart J. 2016;37(29):2315-81.

\section{Publisher's Note}

Springer Nature remains neutral with regard to jurisdictional claims in published maps and institutional affiliations. 